\title{
Determination of the nanoscale dielectric constant by means of a double pass method using electrostatic force microscopy
}

Cite as: J. Appl. Phys. 106, 024315 (2009); https://doi.org/10.1063/1.3182726

Submitted: 13 April 2009. Accepted: 20 June 2009. Published Online: 31 July 2009

C. Riedel, R. Arinero, Ph. Tordjeman, M. Ramonda, G. Lévêque, G. A. Schwartz, D. G. de Oteyza, A. Alegria, and J. Colmenero

\section{ARTICLES YOU MAY BE INTERESTED IN}

Dielectric-constant measurement of thin insulating films at low frequency by nanoscale capacitance microscopy

Applied Physics Letters 91, 243110 (2007); https://doi.org/10.1063/1.2821119

Quantifying the dielectric constant of thick insulators using electrostatic force microscopy Applied Physics Letters 96, 183107 (2010); https://doi.org/10.1063/1.3427362

Nanoscale capacitance microscopy of thin dielectric films

Journal of Applied Physics 104, 024315 (2008); https://doi.org/10.1063/1.2957069

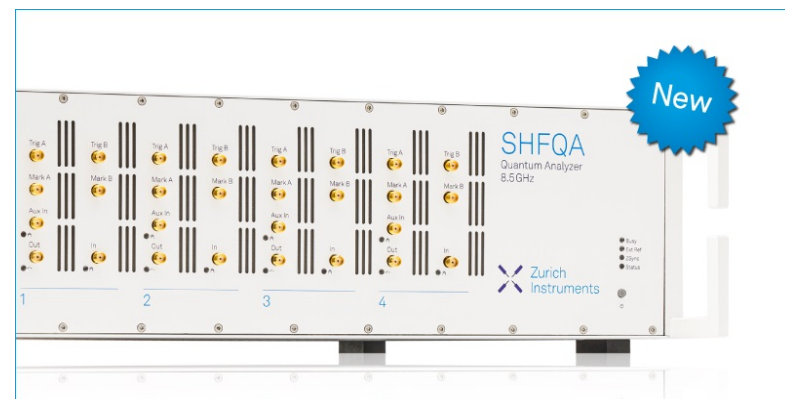

\section{(1)

Meet the next generation of quantum analyzers

- Readout for up to 64 qubits

- Operation at up to $8.5 \mathrm{GHz}$, mixer-calibration-free

- Signal optimization with minimal latency
Find out more

Zurich

Instruments 


\title{
Determination of the nanoscale dielectric constant by means of a double pass method using electrostatic force microscopy
}

\author{
C. Riedel,,${ }^{1,2,3}$ R. Arinero, ${ }^{1, a)}$ Ph. Tordjeman, ${ }^{4}$ M. Ramonda, ${ }^{5}$ G. Lévêque, ${ }^{1}$ G. A. Schwartz, ${ }^{6}$ \\ D. G. de Oteyza, ${ }^{2}$ A. Alegria, ${ }^{3,6}$ and J. Colmenero, ${ }^{2,3,6}$ \\ ${ }_{1}^{1}$ Institut d'Electronique du Sud (IES), UMR CNRS 5214, Université Montpellier II, CC 082, Place E. \\ Bataillon, 34095 Montpellier Cedex, France \\ ${ }^{2}$ Donostia International Physics Center, Paseo Manuel de Lardizabal 4, 20018 San Sebastián, Spain \\ ${ }^{3}$ Departamento de Física de Materiales, Facultad de Química, UPV/EHU, Apartado 1072, 20080 San \\ Sebastián, Spain \\ ${ }^{4}$ Institut de Mécanique des Fluides (IMFT), Université de Toulouse-CNRS, 1 Allée du Professeur Camille \\ Soula, 31400 Toulouse, France \\ ${ }^{5}$ Laboratoire de Microscopie en Champ Proche (LMCP), Centre de Technologie de Montpellier, Université \\ Montpellier II, CC 082, Place E. Bataillon, 34095 Montpellier Cedex, France \\ ${ }^{6}$ Centro de Física de Materiales (CSIC-UPV/EHU)-Materials Physics Center MPC, Edificio Korta, 20018 \\ San Sebastián, Spain
}

(Received 13 April 2009; accepted 20 June 2009; published online 31 July 2009)

\begin{abstract}
We present a method to determine the local dielectric permittivity of thin insulating layers. The measurement is based on the detection of force gradients in electric force microscopy by means of a double pass method. The proposed experimental protocol is simple to implement and does not need any modification of standard commercial devices. Numerical simulations based on the equivalent charge method make it possible to carry out quantification whatever the thickness of film, the radius of the tip, and the tip-sample distance. This method has been validated on a thin $\mathrm{SiO}_{2}$ sample for which the dielectric permittivity at the nanoscale has been characterized in the literature. We also show how we can quantitatively measure the local dielectric permittivity for ultrathin polymer film of poly(vinyl acetate) and polystyrene. (C) 2009 American Institute of Physics.
\end{abstract}

[DOI: 10.1063/1.3182726]

\section{INTRODUCTION}

Dielectric relaxation (DR) has shown to be a very useful, extended, and versatile technique to study dielectric materials, in particular, polymers and other glass formers. ${ }^{1}$ The interaction of electromagnetic radiation with systems having permanent molecular dipoles allows obtaining valuable information about the molecular dynamics of the system under investigation at different time (or frequency) scales. Additionally, DR measurements can be performed at different temperatures or pressures to study the corresponding dependence of the molecular dynamics. In spite of all these promising characteristics, the standard DR has a fundamental limitation due to the lack of spatial resolution. This restriction means, for instance, that the study of self-assembly or nanostructurated polymers is in principle model depending. ${ }^{2}$ Advances in our ability to explore local properties will make possible to gain a deeper understanding of the physics of complex materials. In this context, it is important to develop reliable methods for the determination of micro-/ nanodielectric properties that would have broad impact on the field of material science and particularly to study fundamental physics of nanostructured materials.

Some existing works looking for dielectric characterization at nanoscale are based on using atomic force microscopy (AFM) and, more especially, electrostatic force microscopy

\footnotetext{
a) Author to whom correspondence should be addressed. Electronic mail: richard.arinero@ies.univ-montp2.fr.
}

(EFM). EFM senses electrostatic forces and has been used to image localized charges on surfaces, ${ }^{3}$ dielectric constant variations, ${ }^{4,5}$ and potentials. ${ }^{6}$ In EFM a conducting cantilever allows applying dc or ac bias voltages to the tip. It is generally used to measure the surface potential (Nano-Kelvin) on semiconducting materials. ${ }^{7}$ Recently, Israeloff $e t$ al. ${ }^{8,9}$ used ultrahigh vacuum AFM in order to characterize the complex dielectric permittivity $\left(\varepsilon^{*}(\omega)=\varepsilon^{\prime}-i \varepsilon^{\prime \prime}\right)$ of poly(vinyl acetate) (PVAc) polymer. This experiment was realized by applying an ac bias voltage of variable frequency $(\omega)$. From the in and quadrature phase components of the sensor signal response and using a phenomenological model, they obtained the frequency dependence of $\varepsilon^{\prime}$ and $\varepsilon^{\prime \prime}$ without giving quantitative values for the dielectric permittivity. Other reported works were devoted to the determination of the modulus of the dielectric permittivity without taking into account the possible frequency response of the material. We can mention for instance the works of Krayev et al. ${ }^{4,5}$ related to the study of polymers blend in the form of layer of several microns thickness. The authors showed that an electric contrast could be obtained on EFM images and that such a contrast is related to the variations of the relative dielectric permittivity $\varepsilon_{r}$. They also quantified the value of $\varepsilon_{r}$ in the frame of a simple spherical capacitor model. Unfortunately, this model is appropriate only under certain conditions because it makes the approximation that the thickness of the sample is very large compared to the tip radius and the tip-sample distance. Moreover, dielectric constants of two reference polymers are required to measure a third unknown one. Finally, a different 
approach has been recently proposed by Fumagalli and co-workers. ${ }^{10,11}$ The authors developed the so-called "nanoscale capacitance microscopy," which is based on highresolution measurement of capacitance-distance curves. While a sinusoidal voltage is applied between the AFM tip and the bottom electrode of the sample, the ac current is measured using a state of the art high sensitivity current amplifier. From the sample impedance, the tip-sample capacitance can be obtained according to the distance. Then, it is possible to extract the dielectric permittivity of the sample by fitting the capacitance-distance curve with an appropriate model. The authors proposed an analytical model, of which the validity was proven for film thickness lower than 100 nm. ${ }^{11}$

In this article, we present a method to measure the relative dielectric permittivity of thin films at the nanometer scale by EFM. We propose an experimental protocol based on the detection of the electric force gradient by means of a double pass method. ${ }^{7,12,13}$ The value of $\varepsilon_{r}$ is then obtained by fitting experimental points with an expression of the force gradient calculated by the equivalent charge method (ECM). ${ }^{14-16}$ This model is valid whatever the thickness of the film and the radius of the tip. We will initially detail the double pass method and ECM. Then, we will present how the method has been validated on a thin $\mathrm{SiO}_{2}$ sample for which the dielectric permittivity at nanoscale has been already studied. ${ }^{10,11}$ Finally, the dielectric properties of poly(vinyl acetate) (PVAc) and polystyrene (PS) films are investigated at two different temperatures.

\section{THEORETICAL BACKGROUND}

\section{A. electrostatic force microscopy on the basis of a double pass method}

In this work, EFM operations are made under room conditions in the amplitude-controlled mode (Tapping ${ }^{\circledR}$ ) on the basis of a "double pass method.", "12,13 During the first scan the topography is acquired. The tip is then retracted from the surface morphology by a constant height $H_{\text {lift }}$, also called "lift height," and the amplitude of the tip vibration $\delta z$ is reduced in order to stay in the linear regime (amplitude $\ll$ tip-sample distance). During the second scan, while a potential $V_{\mathrm{dc}}$ is applied to the tip (with the sample holder grounded, Fig. 1), the electrostatic force gradient $\operatorname{grad}_{\mathrm{dc}} F$ is detected. The force gradient is related to the cantilever-tipsample capacitance $C(z)$ by $\operatorname{grad}_{\mathrm{dc}} F=(1 / 2)\left(\partial^{2} C(z) / \partial z^{2}\right) V_{\mathrm{dc}}^{2}$, where $z$ is the tip-sample distance. As shown in Fig. 1, during the first scan, the average tip-sample distance is approximately equal to the amplitude $\left(z_{1} \cong \delta z_{1}\right)$. During the second scan, the distance is the sum of the first scan amplitude $\delta z_{1}$ and the lift height $H_{\text {lift }}\left(z_{2} \cong \delta z_{1}+H_{\text {lift }}\right) . \delta z_{2}$ is the second scan amplitude.

There are two possibilities to detect the local electrostatic force gradient. The first one is to measure directly the resonance frequency shift $\Delta f_{0}$ keeping the phase shift constant. The second possibility is to measure the mechanical phase shift $\Delta \Phi$ at constant driving frequency. If we consider that the cantilever-tip-sample system can be approximated by a spring mass system, the relationships between frequency

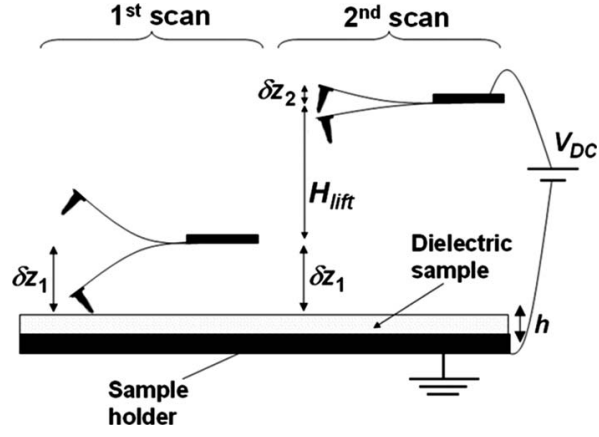

FIG. 1. Principle of EFM microscopy using a double pass method. During the first scan topography is acquired. The tip is then retracted by a constant height $H_{\text {lift }}$ and amplitude is reduced by a factor of about 3. During the second scan, a potential $V_{\mathrm{dc}}$ is applied on the tip and the force gradient is detected.

$\Delta f_{0}$ or phase shifts $\Delta \Phi$ and force gradient $\operatorname{grad}_{\mathrm{dc}} F$, assuming $\operatorname{grad}_{\mathrm{dc}} F \ll k_{c}$ and $\tan \Delta \Phi \cong \Delta \Phi$ (origin at the resonance frequency), can be written as ${ }^{12}$

$$
\begin{aligned}
& \frac{\Delta f_{0}}{f_{0}} \cong-\frac{1}{2} \frac{\operatorname{grad}_{\mathrm{dc}} F}{k_{c}}, \\
& \Delta \Phi \cong-\frac{Q}{k_{c}} \operatorname{grad}_{\mathrm{dc}} F,
\end{aligned}
$$

where $k_{c}$ and $Q$ are the stiffness of the cantilever and the quality factor, respectively. As expected from relations (1) and (2) the curves $\Delta f_{0}\left(V_{\mathrm{dc}}\right)$ and $\Delta \Phi\left(V_{\mathrm{dc}}\right)$ have the parabolic form $-a_{\Delta f_{0}}(z) V_{\mathrm{dc}}^{2}$ and $-a_{\Delta \Phi}(z) V_{\mathrm{dc}}^{2}$, where $a_{\Delta f_{0}}(z)$ and $a_{\Delta \Phi}(z)$ are related to the tip-sample capacitance by the expressions

$$
\begin{aligned}
& a_{\Delta f_{0}}(z)=\frac{f_{0}}{4 k_{c}} \frac{\partial^{2} C(z)}{\partial z^{2}}, \\
& a_{\Delta \Phi}(z)=\frac{Q}{2 k_{c}} \frac{\partial^{2} C(z)}{\partial z^{2}} .
\end{aligned}
$$

If we consider the tip as a cone of half angle $\theta_{0}$, with a spherical apex of radius $R$, attached to the extremity of a cantilever, the total capacitance $C(z)$ is a sum of the apex capacitance $C_{\text {apex }}(z)$, i.e., the local capacitance and the stray capacitance $C_{\text {stray }}(z)$, associated with the tip cone and the cantilever contributions. To correctly model the local capacitance, we must take into account the presence of a thin dielectric layer between the tip and a conductive substrate. For film thicknesses of less than $100 \mathrm{~nm}$, we can refer to the model proposed by Fumagalli et al. ${ }^{10}$ It can be expressed as

$$
C_{\text {apex }}(z)=2 \pi \varepsilon_{0} R \ln \left(1+\frac{R\left(1-\sin \theta_{0}\right)}{z+h / \varepsilon_{r}}\right),
$$

where $h$ and $\varepsilon_{r}$ are the thickness and the relative dielectric constant of the layer, respectively. By fitting the region where the effect of the local capacitance is negligible, they found a stray capacitance of the linear form $C_{\text {stray }}(z)=$ $-b \cdot \Delta z,{ }^{10}$ which does not contribute to the second derivative of the capacitance $\partial^{2} C(z) / \partial z^{2}$ in expressions (3) and (4). In this work we preferred to carry out a numerical modeling of the local capacitance because, as mentioned above, such a 

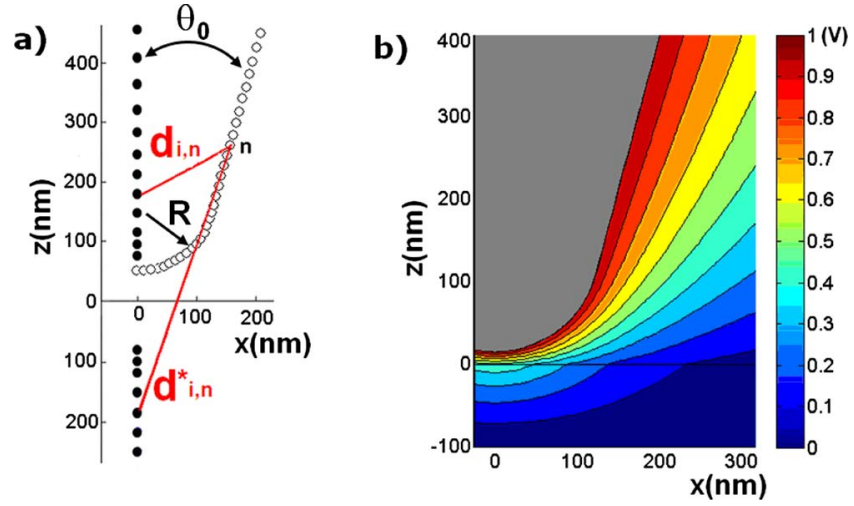

FIG. 2. (Color online) (a) Representation of the charges $(-z>0)$, image charges $(-z<0)$, and test points $(\bigcirc)$ modeling the tip over a metallic plate. (b) The potential created in the air $(z>0)$ and in the dielectric $(z<0)$ by a tip $\left(R=130 \mathrm{~nm}, \theta=15^{\circ}\right)$ in front of a dielectric layer of height $h=100 \mathrm{~nm}$ with a dielectric constant $\varepsilon_{r}=4$. The potential is set to $1 \mathrm{~V}$ at the surface of the tip. The maximum error in one test point is of the order of $1 / 1000$.

model is valid whatever the geometry. In the following section we present the broad outlines of the $\mathrm{ECM},{ }^{14-16}$ which allows to calculate $\varepsilon_{r}$ from the values of the coefficient $a_{\Delta f_{0}}(z)$ or $a_{\Delta \Phi}(z)$.

\section{B. Numerical modeling of the tip-sample capacitance}

In this section, we show how the tip-sample force, gradient, and capacitance can be calculated using the ECM. The advantage of numerical simulation compared to other analytical expression is that the calculated force is exact and allows to work without any restriction about the thickness of the insulating film, the tip radius, and the tip-sample distance. We will initially treat the case of a tip in front of a metallic plate and then we will deduce the force for a system composed by a tip in front of a dielectric layer over a metallic plate.

The case of a system composed of a tip in front of a conductive plane has been treated by Belaidi et al. ${ }^{14}$ The idea of ECM is to find a discrete charge distribution $\left(N_{C}\right.$ charge points $q_{i}$ at a distance $z_{i}$ on the axis $x=0$ ) that will create a given potential $V$ at the tip surface. The conductive plane at a zero potential is created by the introduction of an electrostatic image tip with $-q_{i}$ charges at a distance $-z_{i}$ on the $z$ axis. A good representation of the cone and the tip is given by a finite number of charges (approximately ten charges for the cone and four charges for the tip). The spatial distribution of charges and image charges is shown in Fig. 2(a).

The value of the charges $q_{i}$ is fixed in such way that the $M$ potentials $V_{n}$, with $n=1, \ldots, M$, calculated at test points $n$ at the tip surface are equal to $V$. If we introduce $D_{i, n}$ $=\left(1 / d_{i, n}\right)-\left(1 / d_{i, n}^{*}\right)$ (where $d_{i, n}$ and $d_{i, n}^{*}$ are the distances between the point $n$ and the effective and image charge $i$, respectively), we can express the potential $V_{n}$ as

$$
V_{n}=\sum_{i}^{N_{C}} \frac{D_{i, n} q_{i}}{4 \pi \varepsilon_{0}} .
$$

The best value of $q_{i}$ is obtained using the least mean square method:

$$
\frac{\partial}{\partial q_{i}} \sum_{n}^{M}\left(V_{n}-V\right)^{2}=0 .
$$

Expliciting the derivative of the potential, the system to solve becomes

$$
\sum_{n}^{M}\left(\sum_{i}^{N_{C}} \frac{D_{i, n} q_{i}}{4 \pi \varepsilon_{0}}-V\right) \frac{D_{i, n}}{4 \pi \varepsilon_{0}}=0 .
$$

Then, knowing the charge and image charge distributions, the total electrostatic force acting on the tip and the tip-sample capacitance can be calculated. The coefficient $a_{\Delta f_{0}}(z)\left[\right.$ or $\left.a_{\Delta \Phi}(z)\right]$ is also obtained according to Eq. (3) [or Eq. (4)].

When the system is composed of a tip in front of a dielectric layer placed over a conductive substrate, simulations are more complex. This problem has been treated by Sacha et al. ${ }^{17}$ introducing the Green function formalism and also by Durand. ${ }^{18}$ We consider one charge $q_{i}$ in the air at a distance $z_{i}$ of a dielectric layer of thickness $h$ and of dielectric constant $\varepsilon_{r} . V_{0}^{i}$ and $V_{1}^{i}$ are, respectively, the potentials created by the charge $q_{i}$ in the air and in the dielectric. In order to satisfy the limit conditions $\left(V_{0}^{i}=V_{1}^{i}\right.$ and $\varepsilon_{0}\left(\partial V_{0}^{i} / \partial z\right)$ $=\varepsilon_{O} \varepsilon_{r}\left(\partial V_{1}^{i} / \partial z\right)$ at the air/dielectric interface and $V_{1}^{i}=0$ at the dielectric/substrate interface), we introduce two series of image charges, one created in the conductive substrate and one in the air.

The equivalent potential calculated by ECM in the air results from the source, its image in the dielectric, and the infinite series of image charges in the conductive substrate. One can introduce the "reciprocal distance," $D+$, between a point of coordinate $(\rho, \mathrm{z})$ and the charge $q_{i}$ [i.e., its image $\left.(D-), D \mp=1 / \sqrt{\rho^{2}+\left(z \mp z_{i}\right)^{2}}\right]$, and the reciprocal distance $A$ corresponding to the infinite series of image (A $=\Sigma_{n=0}^{\infty}\left\{k^{n} / \sqrt{\rho^{2}+\left[z+2(n+1) h+z_{i}\right]^{2}}\right\}$, where the constant $k=$ $\left.-\left(\varepsilon_{r}-1 / \varepsilon_{r}+1\right)\right)$. Then, the potential $V_{0}^{i}$ created in the air by one charge $q_{i}$ is expressed as

$$
V_{0}^{i}=\frac{q_{i}}{4 \pi \varepsilon_{0}}\left(D_{+}+k D_{-}-\left(1-k^{2}\right) A\right) .
$$

The potential $V_{1}^{i}$ created in the dielectric is the sum of the two infinite series of images. Introducing the reciprocal distance for the images in the conductive substrate, $B\{B$ $\left.=\Sigma_{n=0}^{\infty}\left[k^{n} / \sqrt{\rho^{2}+\left(z-2 n h-z_{i}\right)^{2}}\right]\right\}$, we obtain

$$
V_{1}^{i}=\frac{q_{i}}{4 \pi \varepsilon_{0}}(1-k)(B-A) .
$$

The value of each $q_{i}$ is then found by solving Eq. (8), inserting the potential $V_{0}^{i}$ calculated after Eq. (9), at each test point representing the tip surface. An example of the potential created in the air and in the dielectric is shown in Fig. 2(b). Knowing the charge and image charge distributions, the total electrostatic force acting on the tip and the tip-sample capacitance can be calculated. The coefficient $a_{\Delta f_{0}}(z)$ [or $\left.a_{\Delta \Phi}(z)\right]$ is obtained according to Eq. (3) [or Eq. (4)]. 


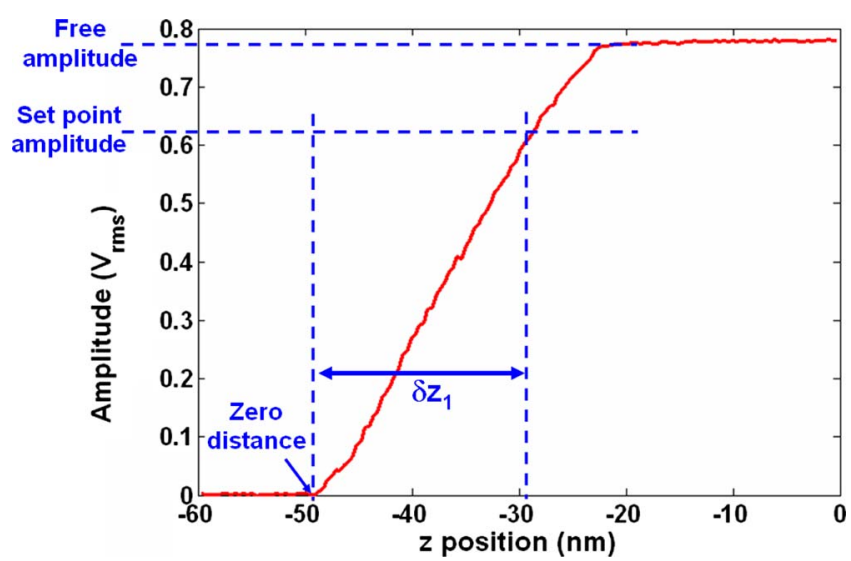

FIG. 3. (Color online) Typical amplitude-distance curve recorded on a stiff sample. The first scan amplitude $\delta z_{1}$ is equal to the difference between the $z$-position corresponding to the set point amplitude and the zero distance.

\section{EXPERIMENTAL PROTOCOL}

The proposed experimental protocol was developed on one single surface position and consists in two steps as detailed below. We point out that although the force gradients can be detected either by measuring the frequency shifts or by measuring the phase shifts, we always chose in this work the measurement of frequency shifts, which offers a better sensitivity. Before describing the experimental procedure, we will discuss a common point to both steps, which is the determination of the actual tip-sample distance.

\section{A. Determination of the actual tip-sample distance}

As shown in the previous section, the lift height $H_{\text {lift }}$ does not correspond to the real tip-sample distance. The average tip-sample distance during the second scan is the sum of the lift height $H_{\text {lift }}$ and the first scan amplitude $\delta z_{1}$. The value of $\delta z_{1}\left(\delta z_{1} \cong 20 \mathrm{~nm}\right)$ is obtained by recording an amplitude-distance curve. A typical experimental curve is shown in Fig. 3; the slope of this curve gives the correspondence between the photodetector rms voltage and the real amplitude. Indeed, if there is no indentation of the tip into the sample, we can consider that amplitude is roughly equivalent to the distance. The zero distance corresponds to the point where amplitude becomes null. The tip-sample distance is calculated as the difference between the z-position of the actuator corresponding to the amplitude set point and the $z$-position corresponding to the zero distance. ${ }^{19}$

\section{B. First step: Determination of the tip radius value by fitting experimental parabolic slopes in the absence of the dielectric film}

This part of the experiment has to be performed on a conductive sample or substrate $\left(h=0, \varepsilon_{r} \rightarrow \infty\right)$. The aim of this step is to extract the actual equivalent value of the tip radius $R . \Delta f_{0}\left(V_{\mathrm{dc}}\right)$ curves are recorded at several lift heights $H_{\text {lift }}$. A parabolic fit allows to extract the experimental coefficients $a_{\Delta f_{0}}(z)$ according to the real tip-sample distance. A value of the radius $R$ is then obtained by fitting the $a_{\Delta f_{0}}(z)$ curve with expression (3) in which the tip-sample capaci-

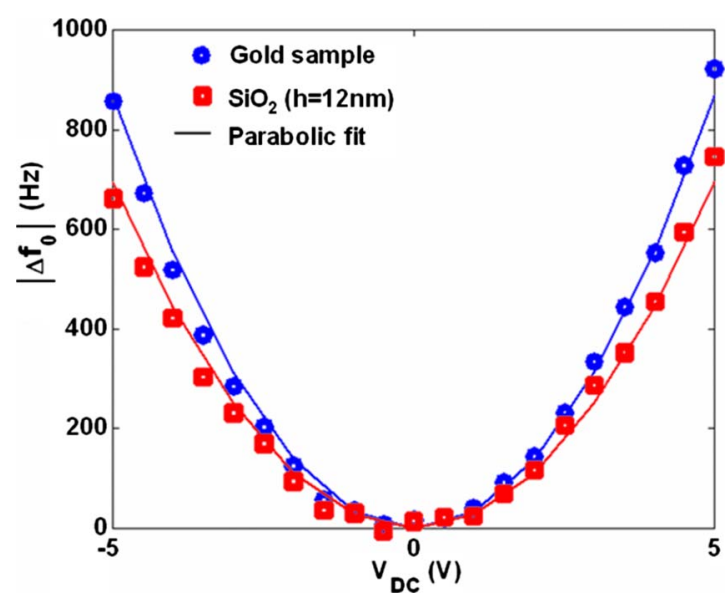

FIG. 4. (Color online) Parabolic profiles of $\Delta f_{0}\left(V_{\mathrm{dc}}\right)$ curves measured on a conductive gold sample (circles) and $\mathrm{a} \mathrm{SiO}_{2} /$ gold sample (squares) with $h_{\mathrm{SiO}_{2}}=12 \mathrm{~nm}$. Both curves were obtained for the same tip-sample distance $z=31 \mathrm{~nm}$. The parabolic fit gives $a_{\Delta f_{0}}=31.7 \mathrm{~Hz} / \mathrm{V}^{2}$ for gold and $a_{\Delta f_{0}}$ $=27.8 \mathrm{~Hz} / \mathrm{V}^{2}$ for $\mathrm{SiO}_{2}$.

tance is calculated using the equivalent charge model (see previous section) without taking into account the insulating layer.

\section{Second step: Determination of the dielectric permittivity by fitting experimental parabolic coefficients in presence of the dielectric film}

The experiment is now preformed with a thin insulating layer of the material under study deposited on the conductive substrate. This second step aims to determine the dielectric permittivity of the investigated layer $\varepsilon_{r} . \Delta f_{0}\left(V_{\mathrm{dc}}\right)$ curves are recorded again at different lift heights $H_{\text {lift }}$ and they are then analyzed in order to extract experimental coefficients $a_{\Delta f_{0}}(z)$ for each lift height. Once $R$ and $h$ are known from previous experiments, we can fit the $a_{\Delta f_{0}}(z)$ curve using expression (3) in which the capacitance is calculated by ECM, thereby we obtain the value of the dielectric permittivity $\varepsilon_{r}$.

\section{EXPERIMENTAL RESULTS}

We developed our method studying the dielectric constant of a model insulating thin layer of $\mathrm{SiO}_{2}$ deposited on a gold substrate. Our samples were similar to those studied by Fumagalli et al. ${ }^{10}$ They were composed of squares of $1 \mu \mathrm{m}$ side deposited by focused ion beam. The average thickness of the $\mathrm{SiO}_{2}$ layers was measured around $12 \mathrm{~nm}$. We used conductive diamond coated tips (Nanosensors ${ }^{\text {TM }}$ CDT-FMR) having a free oscillating frequency $f_{0}=103 \mathrm{kHz}$ and a stiffness $k_{c}=5.9 \mathrm{~N} \mathrm{~m}^{-1} . k_{c}$ was calculated using the so-called thermal tune method ${ }^{20}$ based on the thermal noise measurement. The experiments were realized with a Veeco Enviroscope $^{\mathrm{TM}}$ equipped with a Lakeshore temperature controller. In Fig. 4, we show the $\Delta f_{0}\left(V_{\mathrm{dc}}\right)$ curve obtained on the gold conductive sample in comparison with the curve obtained on the insulating oxide layer. Both curves were acquired at the same tip-sample distance $z=31 \mathrm{~nm}$. We observed that the parabolic profile in the presence of the oxide layer tends "to open" what is revealing a reduction in the local capacitance 


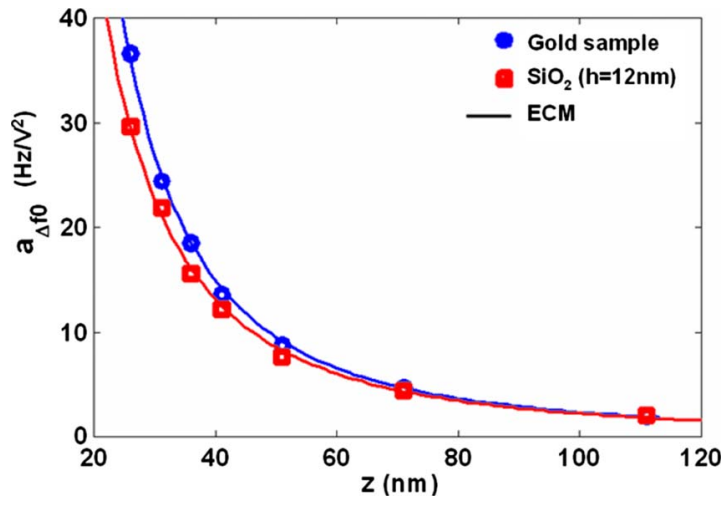

FIG. 5. (Color online) $a_{\Delta f_{0}}(z)$ curves measured on a conductive gold sample (circles) and a $\mathrm{SiO}_{2}$ /gold sample (squares) with $h_{\mathrm{SiO}_{2}}=12 \mathrm{~nm}$. The tip radius $R=105 \pm 4 \mathrm{~nm}$ is obtained from experiments on gold using ECM. Then, by fitting the $\mathrm{SiO}_{2}$ experiments, we calculated the permittivity of the $\mathrm{SiO}_{2}$ insulating layer: $\varepsilon_{r}=4.5 \pm 1.1$.

in accordance with Eq. (3). By fitting these curves using a parabolic function, we obtained $a_{\Delta f_{0}}=31.7 \mathrm{~Hz} / \mathrm{V}^{2}$ for gold and $a_{\Delta f_{0}}=27.8 \mathrm{~Hz} / \mathrm{V}^{2}$ for $\mathrm{SiO}_{2}$.

In Fig. 5, we present the parabolic coefficients $a_{\Delta f_{0}}$ as a function of the real tip-sample distance obtained on gold and $\mathrm{SiO}_{2}$. The fit on gold allows to estimate the value of the effective radius of the tip ( $R=105 \pm 4 \mathrm{~nm}$ in this case). Then, we calculated the value of the dielectric permittivity of the insulating layer by fitting the points obtained on $\mathrm{SiO}_{2}$. We found $\varepsilon_{r}=4.5 \pm 1.1$, which is in agreement with the value obtained by Fumagalli et al. ${ }^{10}$ on the same type of sample. The best-fitting curves were obtained by the least-squares method and the final uncertainties were calculated including uncertainties of all others parameters involved in the calculations.

The second series of experiments was performed on two ultrathin polymer films. PS $\left(M_{w}=70950 \mathrm{~g} / \mathrm{mol}\right)$ and PVAc $\left(M_{w}=83000 \mathrm{~g} / \mathrm{mol}\right)$ were chosen because both the dielectric strength and its temperature dependence are very different for these two polymers. Additionally, the dielectric responses of both polymers have been previously well characterized in the literature. ${ }^{21-25}$ Samples were prepared by spin coating starting from solutions at $1 \%(w / w)$ in toluene. The substrate was composed of a fine gold layer deposited on a glass plate. The small percentage of polymer in solution was selected in order to obtain films with a thickness of about $50 \mathrm{~nm}$ according to Ref. 26. We used in this case standard EFM cantilevers (Nanosensors EFM) having a free oscillating frequency $f_{0}=71.42 \mathrm{kHz}$ and a stiffness $k_{c}$ $=4.4 \mathrm{~N} \mathrm{~m}^{-1}$. The experiments were performed on neat PS and PVAc films at room temperature and at $70{ }^{\circ} \mathrm{C}$ (Figs. 6 and 7). The measured thicknesses of the films were $50 \pm 2 \mathrm{~nm}$ for PS and $50 \pm 3 \mathrm{~nm}$ for PVAc at both room temperature and $70{ }^{\circ} \mathrm{C}$. The thicknesses were determined by $\mathrm{AFM}$, measuring the height difference between the polymer surface and the gold substrate after the films were cut using a sharp steel knife. The accuracy of our measurements does not allow detecting any thermal expansion.

The experimental parabolic coefficients $a_{\Delta f_{0}}(z)$ obtained for PS are shown in Fig. 6. Measurements at room temperature and at $70{ }^{\circ} \mathrm{C}$ are very close indicating a weak tempera-

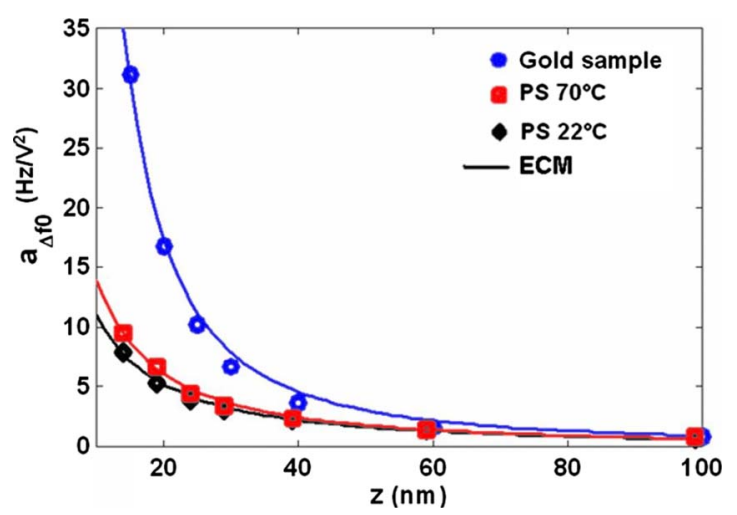

FIG. 6. (Color online) $a_{\Delta f_{0}}(z)$ curves obtained on a $50 \pm 3 \mathrm{~nm}$ PS thin film at $22{ }^{\circ} \mathrm{C}$ (diamond) and $70{ }^{\circ} \mathrm{C}$ (squares) in comparison with the curve obtained on a gold sample (circles). The tip radius $R=32 \pm 2 \mathrm{~nm}$ is obtained from experiments on gold using ECM. Fitting PS parabolic coefficients using ECM, we obtained $\varepsilon_{r}=2.2 \pm 0.2$ at $22{ }^{\circ} \mathrm{C}$ and $\varepsilon_{r}=2.6 \pm 0.3$ at $70{ }^{\circ} \mathrm{C}$.

ture dependence of the dielectric permittivity as expected for this polymer. In addition, there is a big difference between the curve obtained on gold and those obtained on PS. That means that the permittivity of the polymer is rather low. Using the same protocol, we obtained the value of the tip radius $R=32 \pm 2 \mathrm{~nm}$ and the dielectric permittivity of PS at 22 and $70{ }^{\circ} \mathrm{C}: \varepsilon_{r}\left(22{ }^{\circ} \mathrm{C}\right)=2.2 \pm 0.2$ and $\varepsilon_{r}\left(70{ }^{\circ} \mathrm{C}\right)=2.6 \pm 0.3$. The experimental parabolic coefficients obtained for PVAc are shown in Fig. 7. We can note a significant difference between measurements realized at room temperature and at $70{ }^{\circ} \mathrm{C}$, i.e., below and above the glass transition temperature, $T_{g}$. At $70{ }^{\circ} \mathrm{C}$, the PVAc curve approaches the gold curve indicating an important increase of $\varepsilon_{r}$. By applying ECM, we obtained $\varepsilon_{r}\left(22^{\circ} \mathrm{C}\right)=2.9 \pm 0.3$ and $\varepsilon_{r}\left(70{ }^{\circ} \mathrm{C}\right)=8.2 \pm 1.0$ for PVAc. The estimated values for PS and PVAc are in good agreement with the macroscopic ones. ${ }^{21-25}$ The variation observed in the dielectric permittivity of PVAc is related with its strong dipole moment and the fact that PVAc crossed the glass transition temperature at around $38{ }^{\circ} \mathrm{C}$ increasing the chain mobility and therefore the dielectric permittivity. Opposite, PS has a weak dipole moment and its $T_{g}$ is around $105^{\circ} \mathrm{C}$; therefore, little or negligible variation in the dielectric permittivity is expected in this case.

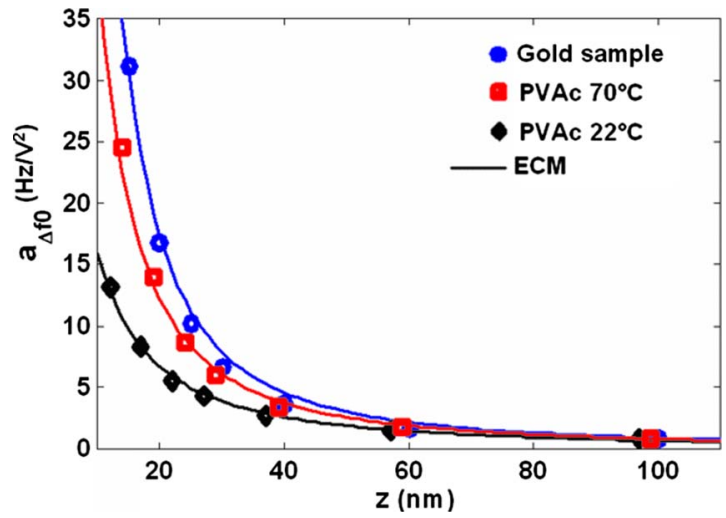

FIG. 7. (Color online) $a_{\Delta f_{0}}(\mathrm{z})$ curves obtained on a $50 \pm 2 \mathrm{~nm}$ PVAc thin film at $22{ }^{\circ} \mathrm{C}$ (diamond) and $70{ }^{\circ} \mathrm{C}$ (squares) in comparison with the curve obtained on a gold sample (circles). The tip radius $R=32 \pm 2 \mathrm{~nm}$ is obtained from experiments on gold using ECM. Fitting PVAc parabolic coefficients using ECM, we obtained $\varepsilon_{r}=2.9 \pm 0.3$ at $22{ }^{\circ} \mathrm{C}$ and $\varepsilon_{r}=8.2 \pm 1.0$ at $70{ }^{\circ} \mathrm{C}$. 


\section{CONCLUSIONS}

We developed a method allowing measuring the dielectric permittivity of a thin film by means of EFM. This method based on the detection of the electrical force gradient by means of a double pass method is simple enough to be implemented with commercial apparatus and the results can be quantified by using the ECM. We proved the feasibility of the method by studying a silicon dioxide film deposited on a gold substrate. This study was validated by a comparison with results recently published by other group using a different method. Further, we applied this method to the study of dielectric properties of polymer thin films. We measured the relative dielectric permittivity of PS and PVAc at two different temperatures and we obtained results in good agreement with those at the macroscopic scale. In perspective, we think that this method could be used to characterize the dielectric properties of polymer blends and of nanocomposite materials at the nanoscale, due to its ability to measure quantitatively $\varepsilon_{r}$ with a high lateral resolution.

\section{ACKNOWLEDGMENTS}

We would like to gratefully acknowledge J. J. Saenz from Universidad Autónoma de Madrid (MoLE group) and Dr. Gabriel Gomila from the Institute for Bioengineering of Catalonia for fruitful discussions and advices. We are also grateful toward María Jesús López Bosque from the "Plataforma de Nanotecnologia" of Barcelona for $\mathrm{SiO}_{2} /$ gold samples preparation. The Donostia Internacional Physics Center (DIPC) financial support is acknowledged. A.A., G.S., and J.C. acknowledge the financial support provided by the Basque Country Government (Grant No. IT-436-07, Depto. Educación, Universidades e Investigación), the Spanish Ministry of Science and Innovation (Grant No. MAT 2007-63681), and the European Community (SOFTCOMP program).

${ }^{1}$ F. Kremer and A. Schönhals, Broadband Dielectric Spectroscopy (Springer-Verlag, Berlin, 2003).
${ }^{2}$ R. Lund, L. Willner, A. Alegria, J. Colmenero, and D. Richter, Macromolecules 41, 511 (2008).

${ }^{3}$ B. D. Terris, J. E. Stern, D. Rugar, and H. J. Mamin, Phys. Rev. Lett. 63, 2669 (1989)

${ }^{4}$ A. V. Krayev and R. V. Talroze, Polymer 45, 8195 (2004).

${ }^{5}$ A. V. Krayev, G. A. Shandryuk, L. N. Grigorov, and R. V. Talroze, Macromol. Chem. Phys. 207, 966 (2006).

${ }^{6}$ Y. Martin, D. W. Abraham, and H. K. Wickramasinghe, Appl. Phys. Lett. 52, 1103 (1988)

${ }^{7}$ P. Girard, M. Ramonda, and D. Saluel, J. Vac. Sci. Technol. B 20, 1348 (2002).

${ }^{8}$ P. S. Crider, M. R. Majewski, J. Zhang, H. Oukris, and N. E. Israeloff, Appl. Phys. Lett. 91, 013102 (2007).

${ }^{9}$ P. S. Crider, M. R. Majewski, J. Zhang, H. Oukris, and N. E. Israeloff, J. Chem. Phys. 128, 044908 (2008).

${ }^{10}$ L. Fumagalli, G. Ferrari, M. Sampietro, and G. Gomila, Appl. Phys. Lett. 91, 243110 (2007).

${ }^{11}$ G. Gomila, J. Toset, and L. Fumagalli, J. Appl. Phys. 104, 024315 (2008).

${ }^{12}$ L. Portes, P. Girard, R. Arinero, and M. Ramonda, Rev. Sci. Instrum. 77, 096101 (2006).

${ }^{13}$ L. Portes, M. Ramonda, R. Arinero, and P. Girard, Ultramicroscopy 107, 1027 (2007).

${ }^{14}$ S. Belaidi, P. Girard, and G. Lévêque, J. Appl. Phys. 81, 1023 (1997).

${ }^{15}$ G. Mesa, E. Dobado-Fuentes, and J. J. Saenz, J. Appl. Phys. 79, 39 (1996).

${ }^{16}$ K. Goto and K. Hane, J. Appl. Phys. 84, 4043 (1998).

${ }^{17}$ G. M. Sacha, E. Sahagun, and J. J. Saenz, J. Appl. Phys. 101, 024310 (2007).

${ }^{18}$ E. Durand, Electrostatique (Masson, Paris, 1966), Vol. 3, p. 233.

${ }^{19}$ During the record of the amplitude-distance curve, the tip can be destroyed. We thus recommend to do it at the end of the experiments. Consequently, the adjustable parameter is the lift height. It can vary from positive to negative values, the minimum value corresponding to the height where the tip is in the contact with the sample. In order to maintain the oscillation of the cantilever in a linear regime, we advise to choose a second scan amplitude of approximately three or four times smaller than $\delta \mathrm{z}_{1}$, so $\delta \mathrm{z}_{2} \approx 6 \mathrm{~nm}$.

${ }^{20}$ J. L. Hutter and J. Bechhoefer, Rev. Sci. Instrum. 64, 1868 (1993).

${ }^{21}$ G. A. Schwartz, E. Tellechea, J. Colmenero, and A. Alegría, J. Non-Cryst. Solids 351, 2616 (2005)

${ }^{22}$ G. A. Schwartz, J. Colmenero, and A. Alegría, Macromolecules 39, 3931 (2006).

${ }^{23}$ G. A. Schwartz, J. Colmenero, and A. Alegría, Macromolecules 40, 3246 (2007).

${ }^{24}$ G. A. Schwartz, J. Colmenero, and A. Alegría, J. Non-Cryst. Solids 353, 4298 (2007).

${ }^{25}$ M. Tyagi, J. Colmenero, and A. Alegría, J. Chem. Phys. 122, 244909 (2005).

${ }^{26}$ D. Hall, P. Underhill, and J. M. Torkelson, Polym. Eng. Sci. 38, 2039 (1998). 